\title{
Independence of the Axioms of Hypergroup over the Group
}

\author{
S. K. Navasardyan
}

\begin{abstract}
The independence of the axioms of hypergroup over the group is proven. The proof is composed of two parts. In the first part, the independence of the axioms $(P 3),(A 1),(A 3),(A 5)$ in the system of axioms of hypergroup over the group is shown by fixing the structural mappings $\Phi$ and $\Xi$. In the same way, in the second part of the proof, the independence of the axioms $(P 1),(P 2),(A 2),(A 4)$ is shown by fixing $\Psi$ and $\Lambda$.
\end{abstract}

Key Words: Hypergroup over the group, axioms, independence Mathematics Subject Classification 2010: 41A60, 68U10

In the eternal memory of my supervisor and senior friend Prof. Samuel Dalalyan, with huge gratitude for the fruitful discussions and enormous impact on this work.

\section{Introduction}

The concept of hypergroup over the group naturally arises when one tries to extend the notion of a quotient group for the case of an arbitrary subgroup. This concept was firstly introduced in [1] and developed in [2] and [3]

Let us emphasize that R. Lal has introduced the concept of $c$-groupoids [5], which coincides with a special case of the hypergroups over the group, namely the unitary hypergroups over the group [4]. The concept of $c$ groupoids has found its applications in the characterization of Tarski monsters [6], in research on right transversals in topological groups [7], and other problems. The concept of hypergroups over the group has arisen independently from the notion of $c$-groupoids.

Also, let us mention that the term "hypergroup" is already used for another concept. Here this concept of hypergroup is not considered, so sometimes we will call the hypergroups over the group shortly hypergroups. 
The further development of hypergroups over the group was done on the basis of the axiomatic system introduced in [3]. This axiomatic system is consistent, since models based on the group theory are constructed for it (see [3]).

In the present paper, the independence of this axiomatic system is proved. We use the following well-known principle. In a consistent axiomatic system, to show the independence of an axiom, it is sufficient to construct a model satisfying all the axioms except the one under consideration. Following this principle, we present eight models for showing the independence of each axiom from others.

In summary, the main result of this paper is the following. A hypergroup over the group is defined as a triple $(M, H, \Omega)$, where $M$ is a set, $H$ is a group and $\Omega=(\Phi, \Psi, \Xi, \Lambda)$ is a system of mappings (see the first section of this paper) which satisfies the conditions $(P 1),(P 2),(P 3),(P 4)$.

Theorem 1 (The main result) The axiomatic system of hypergroup over the group, consisted of $(P 1),(P 2),(P 3)$ and the identities $(A 1),(A 2),(A 3),(A 4),(A 5)$ making up $(P 4)$, is independent.

\section{Definition of the hypergroup over the group}

It is well known that for a given group $G$ and its arbitrary normal subgroup $H$, the quotient group is defined in two steps. First, for any subgroup $S$, the right $S \backslash G$ and the left $G / S$ quotient sets are defined. Then, as $H$ is a normal subgroup, we get $H \backslash G=G / H$, and on this set, a well-defined binary group operation is induced from the group operation of $G$.

In general, when the subgroup $H$ is not necessarily normal, the left and the right quotient sets $H \backslash G$ and $G / H$ are not the same. Moreover, the above-mentioned binary operation induced from the group operation of $G$ is not well-defined. In this case a (right) transversal $M$ of the subgroup $H$ in $G$ is considered, i.e., a subset $M \subset G$ such that $|M \cap H a|=1$ for any $a \in G$. It is proven (see [8]) that the following statements are equivalent.

- $M$ is a (right) transversal of the subgroup $H$ in $G$;

- $M$ is a (right) complementary set of the subgroup $H$ in $G$, i.e., for any $x \in G$, there are unique elements $\alpha \in H, a \in M$ such that $x=\alpha \cdot a$.

Thus, if $M$ is a (right) transversal of the subgroup $H$ in $G$, for any elements $\alpha \in H$ and $a, b \in M$, the elements $\alpha \cdot a, a \cdot b \in G$ are uniquely represented as products of an element from $H$ and an element from $M$ :

$$
a \cdot \alpha={ }^{a} \alpha \cdot a^{\alpha}, \quad a \cdot b=(a, b) \cdot[a, b],
$$

where ${ }^{a} \alpha,(a, b) \in H$ and $a^{\alpha},[a, b] \in M$. 
The concept of the hypergroup over the group arises when considering the triple $(G, H, M)$ and the mapping: ${ }^{1}$

$(\Phi) \Phi: M \times H \rightarrow M, \quad \Phi(a, \alpha)=a^{\alpha}$,

( $\Psi) \Psi: M \times H \rightarrow H, \quad \Psi(a, \alpha)={ }^{a} \alpha$,

$(\Xi) \Xi: M \times M \rightarrow M, \quad \Xi(a, b)=[a, b]$,

( $\Lambda) \Lambda: M \times M \rightarrow H, \quad \Lambda(a, b)=(a, b)$.

It is proven ([3], Theorem 2) that the following conditions are satisfied:

(P1) The mapping $\Xi$ is a binary operation on $M$ such that

(i) any equation $[x, a]=b$ with elements $a, b \in M$ has a unique solution in $M$;

(ii) $(M, \Xi)$ has a left neutral element $o$, i.e., $[o, a]=a$ for any $a \in M$.

(P2) The mapping $\Phi$ is an action of the group $H$ on the set $M$, i.e.,

(i) $\left(a^{\alpha}\right)^{\beta}=a^{\alpha \cdot \beta}$ for any elements $\alpha, \beta \in H$ and for every $a \in M$;

(ii) $a^{\varepsilon}=a$ for each $a \in M$, where $\varepsilon$ is the neutral element of $H$.

(P3) For any $\alpha \in H$, there is an element $\beta \in H$ such that $\alpha={ }^{\circ} \beta$.

$(P 4)$ The following identities $(A 1)-(A 5)$ hold:

$(A 1){ }^{a}(\alpha \cdot \beta)={ }^{a} \alpha \cdot{ }^{a} \beta$,

(A2) $[a, b]^{\alpha}=\left[a^{b} \alpha, b^{\alpha}\right]$,

(A3) $(a, b) \cdot{ }^{[a, b]} \alpha={ }^{a}\left({ }^{b} \alpha\right) \cdot\left(a^{b} \alpha, b^{\alpha}\right)$,

$(A 4)[[a, b], c]=\left[a^{(b, c)},[b, c]\right]$,

$(A 5)(a, b) \cdot([a, b], c)={ }^{a}(b, c) \cdot\left(a^{(b, c)},[b, c]\right)$.

Now let us define the (right) hypergroup over the group. For an arbitrary set $M$, a group $H$ and a system of mappings $\Omega=(\Phi, \Psi, \Xi, \Lambda)$, we call the triple $(M, H, \Omega)$ a (right) hypergroup over the group if the conditions $(P 1)-(P 4)$ are satisfied. Such a hypergroup over the group is denoted by $M_{H}$.

In this paper, we will also use the notion of triviality of the structural mappings $\Phi, \Psi, \Xi, \Lambda$. The mappings $\Phi, \Psi, \Xi, \Lambda$ are called trivial if, respectively,

$(\operatorname{Tr} \Phi) \Phi(a, \alpha)=a$ for any $a \in M, \alpha \in H$

$(\operatorname{Tr} \Psi) \Psi(a, \alpha)=\alpha$ for any $a \in M, \alpha \in H$,

\footnotetext{
${ }^{1}$ Further in this paper, we will often use these notations for the mappings $\Phi, \Psi, \Xi, \Lambda$.
} 
$(\operatorname{Tr} \Xi) \Xi(a, b)=o$ for any $a, b \in M$ and, consequently, $M=\{o\}$,

$(\operatorname{Tr} \Lambda) \Lambda(a, b)=\varepsilon$ for any $a, b \in M$.

Remark 1 Note that in [4], the triviality of the mapping $\Lambda$ is defined as $\Lambda(a, b)=\Lambda(o, o)$ (for any $a, b \in M)$. In this paper, we will need only its special case when $\Lambda(o, o)=\varepsilon$. Thus there will be no misconceptions if here we call $\Lambda$ trivial in only this special case.

\section{The independence of the axioms $(P 3),(A 1)$, $(A 3)$ and $(A 5)$}

In this section, by fixing the mappings $\Phi, \Xi$ and varying the set $M$, the group $H$ and the mappings $\Psi, \Lambda$, we construct models showing the independence (in the axiomatic system of hypergroup over the group) of the axioms (P3), $(A 1),(A 3)$ and $(A 5)$.

Consider an arbitrary set $M$ and a group $H$ with the neutral element $\varepsilon$. Let the mapping $\Phi: M \times H \rightarrow M$ be trivial $(\operatorname{Tr} \Phi)$ and fix the binary operation $\Xi: M \times M \rightarrow M$ such that $(M, \Xi)$ is a group with the neutral element $o \in M$.

It turns out that the fixation of $\Phi$ and $\Xi$ indicated above is sufficient for some axioms to be satisfied. More precisely, the following lemma holds.

Lemma 1 For an arbitrary set $M$, a group $H$ and mappings $\Phi, \Psi, \Xi, \Lambda$, if $(M, \Xi)$ is a group and $\Phi$ is trivial, the conditions $(P 1),(P 2),(A 2),(A 4)$ are satisfied. Moreover, the identities $(A 1),(A 3),(A 5)$ turn into the identities:

$(A 1)^{\prime}{ }^{a}(\alpha \cdot \beta)={ }^{a} \alpha \cdot{ }^{a} \beta$,

$(A 3)^{\prime}(a, b) \cdot{ }^{[a, b]} \alpha={ }^{a}\left({ }^{b} \alpha\right) \cdot(a, b)$,

$(A 5)^{\prime}(a, b) \cdot([a, b], c)={ }^{a}(b, c) \cdot(a,[b, c])$,

respectively, for any $a, b, c \in M, \alpha, \beta \in H$.

Thus, according to Lemma 1, for the fixed mappings $\Phi, \Xi$, the axioms $(P 1),(P 2),(A 2),(A 4)$ are satisfied. Hence, for the independence of any axiom $X \in\{(P 3),(A 1),(A 3),(A 5)\}$ it is sufficient to choose a set $M$, a group $H$ and mappings $\Psi, \Lambda$ such that all the axioms $(P 3),(A 1),(A 3),(A 5)$ are satisfied except the axiom $X$.

For this, let us consider the set $T_{H}$ composed of all mappings $H \rightarrow H$ and the function composition as a binary operation on it (the full transformation semigroup of $H$ ). Let $\bar{\Psi}$ be the following mapping:

$$
\bar{\Psi}: M \rightarrow T_{H}, \quad \bar{\Psi}(a)=\Psi_{a}, \quad \text { where } \quad \Psi_{a}: H \rightarrow H, \quad \alpha \mapsto{ }^{a} \alpha .
$$


Theorem 2 allows us to describe for each axiom $(P 3),(A 1),(A 3),(A 5)$ a family of models showing the independence of that axiom.

Theorem 2 Let $M$ be an arbitrary set, $H$ be a group, $\Omega=(\Phi, \Psi, \Xi, \Lambda)$ be a system of mappings such that $\Phi$ is trivial and $(M, \Xi)$ is a group. In that case,

1) if $\Lambda$ is trivial, then for the fulfillment of the axioms $(A 1),(A 3),(A 5)$ and non-fulfillment of the axiom (P3), it is necessary and sufficient that $\bar{\Psi}$ be an antihomomorphism and $\bar{\Psi}(a)$ be a non-surjective homomorphism for any $a \in M$;

2) if $\Lambda$ is trivial and $H$ is a finite group, then for the fulfilment of the axioms $(P 3),(A 3),(A 5)$ and non-fulfilment of the axiom $(A 1)$, it is necessary and sufficient that $\bar{\Psi}$ be an antihomomorphism, $\bar{\Psi}(a)$ be such a bijective function that $\bar{\Psi}(a)(\varepsilon)=\varepsilon$ for any $a \in M$, and $\bar{\Psi}(a)$ be $a$ non-homomorphic function for an element $a \in M$;

3) if $\Lambda$ is trivial and $H$ is a finite group, then for the fulfilment of the axioms $(P 3),(A 1),(A 5)$ and non-fulfillment of the axiom $(A 3)$, it is necessary and sufficient that $\bar{\Psi}$ be a non-antihomomorphism, $\bar{\Psi}(a)$ be a homomorphism for any $a \in M$, and $\bar{\Psi}(o)$ be a bijection;

4) if $\Psi$ is trivial then for the fulfilment of the axioms $(P 3),(A 1),(A 3)$ and non-fulfilment of the axiom (A5), it is necessary and sufficient that elements $\Lambda(a, b)$ be in the center $Z(H)$ of the group $H$ for any $a, b \in M$, and the following identity not be satisfied for some $a, b, c \in M$ :

$$
(A 5)^{\prime \prime} \quad(a, b) \cdot([a, b], c)=(b, c) \cdot(a,[b, c]) .
$$

Proof. Since in the points 1) - 3) of the theorem, the triviality of $\Lambda$ is present as a condition, let us first assume, that $\Lambda$ is trivial. Then $(P 3),(A 1),(A 3),(A 5)$ turn, respectively, into the following conditions:

$(\bar{\Psi} P 3) \bar{\Psi}(o): H \rightarrow H$ is a surjective mapping;

$(\bar{\Psi} A 1) \bar{\Psi}(a): H \rightarrow H$ is a homomorphism for any $a \in M$;

$(\bar{\Psi} A 3) \bar{\Psi}: M \rightarrow T_{H}$ is an antihomomorphism, i.e., $\bar{\Psi}([a, b])=\bar{\Psi}(b) \circ \bar{\Psi}(a)$ for any $a, b \in M$;

$(\bar{\Psi} A 5) \bar{\Psi}(a)(\varepsilon)=\varepsilon$ for any $a \in M$.

Let us prove the points 1) -3 ) of Theorem 2.

1) Suppose the conditions $(A 1),(A 3),(A 5)$ be satisfied and $(P 3)$ not. Then, according to $(\bar{\Psi} A 3)$ and $(\bar{\Psi} A 1), \bar{\Psi}$ is an antihomomorphism and $\bar{\Psi}(a)$ 
is a homomorphism for any $a \in M$. It remains to show that $\bar{\Psi}(a)$ is not surjective for any $a \in M$. Indeed, if for an element $a \in M$ the mapping $\bar{\Psi}(a)$ is surjective, then from $(\bar{\Psi} A 3)$ and the fact that $(M, \Xi)$ is a group, we get $\bar{\Psi}(a)=\bar{\Psi}([o, a])=\bar{\Psi}(a) \circ \bar{\Psi}(o)$, which means $\bar{\Psi}(a) \circ \bar{\Psi}(o)$ is surjective, hence, $\bar{\Psi}(o)$ is also surjective. On the other hand, $(P 3)$ is not satisfied.

$2)$ Let the axioms $(P 3),(A 3),(A 5)$ be satisfied and $(A 1)$ not. Then, according to $(\bar{\Psi} A 3)$ and $(\bar{\Psi} A 5), \bar{\Psi}$ is an antihomomorphism and for any $a \in M$, the mapping $\bar{\Psi}(a): H \rightarrow H$ fixes $\varepsilon$. Since the condition $(\bar{\Psi} A 1)$ is not satisfied, there exists an element $a \in M$ such that $\bar{\Psi}(a)$ is not a homomorphism. It remains to show that $\bar{\Psi}(a)$ is bijective for any $a \in M$. Indeed, notice that from $(\bar{\Psi} P 3)$ and the finiteness of $H$, it follows that $\bar{\Psi}(o)$ is bijective. On the other hand, due to $(\bar{\Psi} A 3)$ we have

$$
\bar{\Psi}(o)=\bar{\Psi}([o, o])=\bar{\Psi}(o) \circ \bar{\Psi}(o) .
$$

Hence,

$$
1_{H}=\bar{\Psi}(o)=\bar{\Psi}\left(\left[a, a^{\prime}\right]\right)=\bar{\Psi}\left(a^{\prime}\right) \circ \bar{\Psi}(a),
$$

where $a^{\prime}$ is the inverse element of $a$ in the group $(M, \Xi)$ and $1_{H}$ is the identity mapping of the set $H$ (it can be shown by the same way, that $\bar{\Psi}\left(a^{\prime}\right)$ is also the right inverse element of $\bar{\Psi}(a))$. Therefore, $\bar{\Psi}(a)$ is bijective for any $a \in M$.

$3)$ Since $H$ is a finite group, the surjectivity of $\bar{\Psi}(o)$ implies its bijectivity.

Now let us prove the point 4 ).

4) Since $\Psi$ is trivial, we get that $(P 3)$ is satisfied and $(A 1)$ becomes a tautology. Notice that $(A 3)$ turns into the following identity:

$$
(a, b) \cdot \alpha=\alpha \cdot(a, b)
$$

for any $\alpha \in H, a, b \in M$, which is equivalent to the condition $\Lambda(M \times M) \subset$ $Z(H)$. It remains to notice that $(A 5)$ turns into $(A 5)^{\prime \prime}$.

Thus, Theorem 2 gives a possibility to construct models showing the independence of each of $(P 3),(A 1),(A 3),(A 5)$ axioms. Indeed, for constructing the models showing the independence of the axioms $(P 3),(A 1),(A 3)$, it is sufficient to fix the mapping $\Lambda$ as trivial and choose the mapping $\bar{\Psi}: M \rightarrow T_{H}$ in a suitable way. For constructing a model showing the independence of the axiom $(A 5)$, it is sufficient to fix the mapping $\Psi$ as trivial and choose the mapping $\Lambda: M \times M \rightarrow H$.

The model showing the independence of $(P 3)$.

Let us fix the mapping $\Lambda$ as trivial. Then, according to the point 1 ) of Theorem 2, for constructing the model showing the independence of the axiom (P3), it is sufficient to choose a set $M$, a group $H$ and an antihomomorphism $\bar{\Psi}: M \rightarrow T_{H}$ such that $\bar{\Psi}(a)$ be a non-surjective homomorphism for any $a \in M$. 
Let $M$ be an arbitrary set and $H$ be an arbitrary non-trivial group. Consider the mapping $f: H \rightarrow H, f(\alpha)=\varepsilon$ for any $\alpha \in H$, and take

$$
\bar{\Psi}: M \rightarrow T_{H}, \quad \bar{\Psi}(a)=f \quad \text { for any } a \in M .
$$

The model showing the independence of $(A 1)$.

Let us fix the mapping $\Lambda$ as trivial. Then, according to the point 2) of Theorem 2, in order to show the independence of the axiom $(A 1)$, it is sufficient to choose a set $M$, a finite group $H$ and an antihomomorphism $\bar{\Psi}: M \rightarrow T_{H}$ such that $\bar{\Psi}(a)$ be a bijection, $\bar{\Psi}(a)(\varepsilon)=\varepsilon$ for any $a \in M$ and there exists an element $a \in M$ such that $\bar{\Psi}(a)$ is not a homomorphism.

It is not difficult to see that $M$ must have at least two elements. Take $M=\{o, a\}$ and let $o$ be the neutral element of the group $(M, \Xi)$. Also, using the antihomomorphity of $\bar{\Psi}$ and the bijectivity of $\bar{\Psi}(o)$, we get $\bar{\Psi}(o)=1_{H}$. On the other hand, $\bar{\Psi}(a)$ can not be a homomorphism, must be a bijection and must fix the neutral element $\varepsilon$ of the group $H$. If $|H| \leq 3$, there is no such mapping $\bar{\Psi}(a)$ since in this case all bijections fixing the element $\varepsilon$ are homomorphisms.

Consider the cyclic group of order four $H=\left\{\varepsilon, \alpha, \alpha^{2}, \alpha^{3}\right\}$ and the following mapping:

$$
f: H \rightarrow H, \quad f(\varepsilon)=\varepsilon, f(\alpha)=\alpha^{2}, f\left(\alpha^{2}\right)=\alpha, f\left(\alpha^{3}\right)=\alpha^{3} .
$$

It remains to take $\bar{\Psi}(a)=f$ and check that the obtained mapping $\bar{\Psi}: M \rightarrow$ $T_{H}$ is an antihomomorphism.

The model showing the independence of $(A 3)$.

Let us fix the mapping $\Lambda$ as trivial. Then, according to the point 3) of Theorem 2, for showing the independence of the axiom $(A 3)$, it is sufficient to choose a set $M$, a finite group $H$ and a mapping $\bar{\Psi}: M \rightarrow T_{H}$ such that $\bar{\Psi}(a)$ be a homomorphism for any $a \in M, \bar{\Psi}(o)$ be a bijection and $\bar{\Psi}$ not be an antihomomorphism.

Let $M$ be an arbitrary set, $H$ be an arbitrary non-trivial finite group and $f: H \rightarrow H$ be a non-trivial automorphism. It remains to take $\bar{\Psi}(a)=f$ for any $a \in M$.

The model showing the independence of $(A 5)$.

Let us fix the mapping $\Psi$ as trivial. Then, according to the point 4) of Theorem 2, for showing the independence of the axiom $(A 5)$, it is sufficient to choose a set $M$, a group $H$ and a mapping $\Lambda$ such that $\Lambda(M \times M) \subset Z(H)$ and $(A 5)^{\prime \prime}$ is not satisfied for some elements $a, b, c \in M$.

For $(A 5)^{\prime \prime}$ to be not satisfied, we must choose $H$ and $M$ with at least two elements. Consider the cyclic group of the second order $H=\{\varepsilon, \alpha\}$ and the following mapping:

$$
\Lambda: M \times M \rightarrow H, \quad \Lambda(o, o)=\varepsilon, \Lambda(a, b)=\alpha
$$

for any $a, b \in M$ such that $a \neq o$ or $b \neq o$. Then $\Lambda(M \times M)=H=Z(H)$ and $(A 5)^{\prime \prime}$ does not hold for $a=b=o, c \neq o$. 


\section{The independence of the axioms $(P 1),(P 2)$, $(A 2)$ and $(A 4)$}

For showing the independence of the axioms $(P 1),(P 2),(A 2),(A 4)$, consider an arbitrary set $M$, a group $H$ with the neutral element $\varepsilon$, and fix the mappings $\Psi: M \times H \rightarrow H$ and $\Lambda: M \times M \rightarrow H$ as trivial.

It turns out that the trivialities of $\Psi$ and $\Lambda$ are sufficient for some axioms of the hypergroup to be satisfied. More precisely, the following lemma holds.

Lemma 2 For an arbitrary set $M$, a group $H$ and mappings $\Phi, \Psi, \Xi, \Lambda$ where $\Psi$ and $\Lambda$ are trivial, the axiom $2^{2}(P 3),(A 1),(A 3),(A 5)$ are satisfied. Moreover, the identities $(A 2)$ and $(A 4)$ turn into the following identities:

- $(A 2)^{\prime} \quad[a, b]^{\alpha}=\left[a^{\alpha}, b^{\alpha}\right]$,

- $(A 4)^{\prime} \quad[[a, b], c]=\left[a^{\varepsilon},[b, c]\right]$.

Thus, according to Lemma2, for showing the independence of each of the axioms $(P 1),(P 2),(A 2),(A 4)$, it is sufficient to choose a set $M$, a group $H$ and mappings $\Phi$ and $\Xi$ such that $(P 1),(P 2),(A 2),(A 4)$ are satisfied except the axiom under the consideration. For this purpose, consider the full transformation semigroup of $M T_{M}=\{f: M \rightarrow M\}$ and define the following mapping:

$$
\bar{\Phi}: H \rightarrow T_{M}, \quad \bar{\Phi}(\alpha)=\Phi_{\alpha} \text {, where } \Phi_{\alpha}: M \rightarrow M, a \mapsto a^{\alpha} .
$$

Theorem 3 Let $M$ be an arbitrary set, $H$ be a group and $\Omega=(\Phi, \Psi, \Xi, \Lambda)$ be a system of mappings such that $\Psi$ and $\Lambda$ are trivial. In that case,

1) if $(M, \Xi)$ is a group, then for the fulfilment of the axioms $(P 1),(A 2),(A 4)$ and non-fulfilment of $(P 2)$, it is necessary and sufficient that $\bar{\Phi}(\alpha):(M, \Xi) \rightarrow(M, \Xi)$ be a homomorphism for any $\alpha \in H$, $\bar{\Phi}(\varepsilon)$ be the identity mapping of $M$ and $\bar{\Phi}$ not be a homomorphism;

2) if $(M, \Xi)$ is a group, then for the fulfilment of the axioms $(P 1),(P 2),(A 4)$ and non-fulfilment of the axiom (A2), it is necessary and sufficient that $\bar{\Phi}$ be a homomorphism, $\bar{\Phi}(\varepsilon)$ be the identity mapping of $M$ and $\Phi(\alpha)$ not be a homomorphism for an element $\alpha \in H$;

3) if $\Phi$ is trivial, then for the fulfilment of the axioms $(P 2),(A 2),(A 4)$ and non-fulfilment of the axiom $(P 1)$, it is necessary and sufficient that the binary operation $\Xi$ be associative but $(M, \Xi)$ not be a group;

4) if $\Phi$ is trivial and $(P 1)$ holds, then for the fulfilment of the axioms $(P 2),(A 2)$ and non-fulfillment of the axiom (A4), it is necessary and sufficient that $(M, \Xi)$ not be a group.

\footnotetext{
${ }^{2}$ In order to formulate $(P 3)$, there must be an element $o \in M$ fixed in $M$. In Lemma 2 . we will just fix this element without any assumptions about it.
} 
Proof. To prove first two points, assume that $(M, \Xi)$ is a group with the neutral element $o$. Then the axiom $(P 1)$ is satisfied. It is not difficult to see that $(A 2)$ and $(A 4)$ are equivalent, respectively, to

$(\Xi A 2)$ The mapping $\bar{\Phi}(\alpha):(M, \Xi) \rightarrow(M, \Xi), a \mapsto a^{\alpha}$ is a group homomorphism for any $\alpha \in H$;

$(\Xi A 4) \quad \bar{\Phi}(\varepsilon)$ is the identity mapping of $M$, i.e., $a^{\varepsilon}=a$ for any $a \in M$.

Note also that $(P 2)(i)$ is equivalent to $\bar{\Phi}$ being homomorphic and $(P 2)(i i)$ is the same as ( $\Xi A 4)$. Hence, 1$)$ and 2) hold.

Now assume that $\Phi$ is trivial. Then $(P 2)$ holds, $(A 2)$ turns into a tautology and $(A 4)$ turns into the assosiativity property of the binary operation $\Xi$. On the other hand, it is well known that $(M, \Xi)$ is a group if and only if the operation $\Xi$ is associative and satisfies the condition $(P 1)$. From this fact and (P2), (A2), the proof of 3) and 4) follows.

Thus, Theorem 3 gives a possibility to construct models showing the independence of each of the axioms $(P 1),(P 2),(A 2),(A 4)$.

The model showing the independence of $(P 1)$. Fix the mapping $\Phi$ as trivial. To satisfy the conditions of the point 3) of Theorem 3 , one can take $M=\{o, a\}$ and $\Xi(x, y)=y$ for any $x, y \in M$.

Remark 2 The following question may arise: are parts $(P 1)(i)$ and $(P 1)(i i)$ of the axiom (P1) independent as individual axioms in the hypergroup axiomatic system? Notice that in order to show the independence of $(P 1)(i)$ and $(P 1)(i i)$, it is sufficient to show the independence of the axiomatic system $\mathcal{X}=\{(P 1)(i),(P 1)(i i),(A S \Xi)\}$, where $(A S \Xi)$ is the axiom of the associativity of the binary operation $\Xi$. It is well known that $\mathcal{X}$ is equivalent to the axiomatic system of the group theory and is independent.

The model showing the independence of $(P 2)$. Let $\Xi$ be such an operation that $(M, \Xi)$ is a group with the neutral element $o$.

According to the point 1) of the Theorem 3, $M$ and $H$ need to have at least two elements. Consider a non-trivial group $H$, the set $M=\{o, a\}$ and the mapping $f: M \rightarrow M$, defined by $f(x)=o$. Take

$$
\bar{\Phi}: H \rightarrow T_{M}, \quad \bar{\Phi}(\varepsilon)=1_{M}, \quad \bar{\Phi}(\alpha)=f
$$

for any $\alpha \in H, \alpha \neq \varepsilon$. Then $\Phi(\alpha)$ is a homomorphism for any $\alpha \in H$ and $\bar{\Phi}$ is not a homomorphism, as for any $\alpha \neq \varepsilon$,

$$
\bar{\Phi}\left(\alpha \cdot \alpha^{-1}\right)=\bar{\Phi}(\varepsilon)=1_{M} \neq f=f \circ f=\bar{\Phi}(\alpha) \circ \bar{\Phi}\left(\alpha^{-1}\right) .
$$

Remark 3 Here we have shown the independence of $(P 2)$ in the case when $(P 2)(i i)$ is satisfied and $(P 2)(i)$ is not. It turns out that $(P 2)(i i)$ can be implied from $(P 2)(i)$ and the rest of the hypergroup axioms. More precisely, the following lemma holds. 
Lemma 3 If the conditions $(P 1),(P 2)(i)$ and $(A 4)$ are satisfied, then (P2) (ii) is also satisfied.

Proof. By taking $b=o$ in $(A 4)$ and using $(P 1)(i i)$, we get

$$
[[a, o], c]=\left[a^{(o, c)},[o, c]\right]=\left[a^{(o, c)}, c\right] .
$$

Hence, according to $(P 1)(i)$, we get $[a, o]=a^{(o, c)}$ for any $a, c \in M$.

Take $c=o$ and denote $\theta=(o, o)$. Then $[a, o]=a^{\theta}$ for any $a \in M$. On the other hand, according to $(P 2)(i)$, we have $\left(a^{\theta}\right)^{\varepsilon}=a^{\theta \cdot \varepsilon}=a^{\theta}$. Therefore $[a, o]^{\varepsilon}=[a, o]$ for any $a \in M$. It remains to notice that according to $(P 1)(i)$ for any $b \in M$, there exists a (unique) element $a \in M$ such that $[a, o]=b$. Hence, $b^{\varepsilon}=b$ for any $b \in M$.

The model showing the independence of $(A 2)$. Here we also consider such a binary operation $\Xi$ that $(M, \Xi)$ is a group with the neutral element $o$. According to the point 2) of Theorem 3, $M$ and $H$ need to have more than one element.

Let us take $H$ and $(M, \Xi)$ isomorphic (in the simplest case the cyclic group of the second order). Fix an isomorphism $f: H \rightarrow M$ and consider the mapping

$$
\bar{\Phi}: H \rightarrow S_{M} \subset T_{M}, \quad \bar{\Phi}(\alpha)=R_{f(\alpha)},
$$

where $R_{a} \in S_{M}$ is the right translation of the group $(M, \Xi)$ with the element $a \in M$, i.e.,

$$
R_{a}: M \rightarrow M, \quad R_{a}(b)=[b, a] .
$$

Since $f$ is a homomorphism and $R_{[a, b]}=R_{a} \circ R_{b}$ for any $a, b \in M$, it follows that $\bar{\Phi}$ is also a homomorphism:

$$
\bar{\Phi}(\alpha \cdot \beta)=R_{f(\alpha \cdot \beta)}=R_{[f(\alpha), f(\beta)]}=R_{f(\alpha)} \circ R_{f(\beta)}=\bar{\Phi}(\alpha) \circ \bar{\Phi}(\beta) .
$$

It is clear that $\bar{\Phi}(\varepsilon)=R_{f(\varepsilon)}=R_{o}=1_{M}$.

It remains to note that the mapping $\bar{\Phi}(\alpha)=R_{f(\alpha)}$ is not a homomorphism for any element $\alpha \in H, \alpha \neq \varepsilon$.

The model showing the independence of $(A 4)$. Fix the mapping $\Phi$ as trivial. According to the point 4$)$ of Theorem 3 , a groupoid $(M, \Xi)$ needs to have at least three elements. Take $M=\{o, a, b\}$ and consider $\Xi$ with the following table:

\begin{tabular}{|c|c|c|c|}
\hline$\Xi$ & $o$ & $a$ & $b$ \\
\hline$o$ & $o$ & $a$ & $b$ \\
\hline$a$ & $b$ & $o$ & $a$ \\
\hline$b$ & $a$ & $b$ & $o$ \\
\hline
\end{tabular}

It is not difficult to see that $(P 1)$ holds. At the same time, $(M, \Xi)$ is not a group since $\Xi$ is not associative:

$$
[[a, a], b]=[o, b]=b \neq o=[a, a]=[a,[a, b]] .
$$




\section{References}

[1] S. Dalalyan, On hypergroups, prenormal subgroups, and the simplest groups, Abstracts of the Conference Dedicated to 90th Anniversary of M.M. Jrbashyan, Yerevan, 2008, pp. 12-14.

[2] S. Dalalyan, Hypergroups over the group and extensions of a group, Abstracts of the Second International Conference of Mathematics in Armenia, Tsaghkadzor, 2013, pp. 111-112.

[3] S. Dalalyan, Hypergroups over the group and generalizations of Schreier's theorem on group extensions, preprint arXiv:1403.6134, 2014.

[4] S. Dalalyan and S. Navasardyan, On Unitary Hypergroups over the Group, Lobachevskii J. Math., 40 (2019), no. 8, pp. 1045-1057. https://doi.org/10.1134/s1995080219080079

[5] R. Lal, Transversals in groups, J. Algebra, 181 (1996), no. 1, pp. 70-81.

[6] R. Lal and R. Shukla, A characterization of Tarski monsters, Indian J. Pure Appl. Math., 36 (2005), no. 12, pp. 673-678.

[7] R. Lal and R. Shukla, Transversals in non-discrete groups, Proc. Indian Acad. Sci. (Math. Sci.), 115 (2005), no. 4, pp. 429-435. https://doi.org/10.1007/bf02829804

[8] J. J. Rotman, An introduction to the theory of groups, SpringerVerlag, New York, GTM, 148 (1995). https://doi.org/10.1007/978-14612-4176-8

Shant K. Navasardyan

Faculty of Mathematics and Mechanics,

Yerevan State University,

1 Alex Manoogian, Yerevan, 0025, Republic of Armenia.

navasardyanshant@gmail.com

Please, cite to this paper as published in

Armen. J. Math., V. 13, N. 12(2021), pp. 111

https://doi.org/10.52737/18291163-2021.13.12-1-11 